\title{
Exploiting Global Knowledge to Achieve Self-Tuned Congestion Control for $k$-Ary $n$-Cube Networks
}

\author{
Mithuna Thottethodi, Member, IEEE, Alvin R. Lebeck, Senior Member, IEEE, and \\ Shubhendu S. Mukherjee, Member, IEEE
}

\begin{abstract}
Network performance in tightly-coupled multiprocessors typically degrades rapidly beyond network saturation. Consequently, designers must keep a network below its saturation point by reducing the load on the network. Congestion control via source throttling - a common technique to reduce the network load—prevents new packets from entering the network in the presence of congestion. Unfortunately, prior schemes to implement source throttling either lack vital global information about the network to make the correct decision (whether to throttle or not) or depend on specific network parameters, or communication patterns. This paper presents a global-knowledge-based, self-tuned, congestion control technique that prevents saturation at high loads across different communication patterns for $k$-ary $n$-cube networks. Our design is composed of two key components. First, we use global information about a network to obtain a timely estimate of network congestion. We compare this estimate to a threshold value to determine when to throttle packet injection. The second component is a self-tuning mechanism that automatically determines appropriate threshold values based on throughput feedback. A combination of these two techniques provides high performance under heavy load, does not penalize performance under light load, and gracefully adapts to changes in communication patterns.
\end{abstract}

Keywords-Interconnection networks, wormhole, $k$-ary $n$-cubes, congestion control, global information, self-tuning.

\section{INTRODUCTION}

$\mathrm{T}$ IGHTLY-COUPLED multiprocessors provide the performance and ease of programming necessary for many commercial and scientific applications. Their interconnection networks provide the low latency and high bandwidth communication required for a variety of workloads. The advent of multiprocessor systems built with highly aggressive, out-of-order, and speculative microprocessors, simultaneous multithreaded processors [10], and chip multiprocessors [8], promises to dramatically increase the offered load on such multiprocessor networks. Unfortunately, most multiprocessor networks suffer from tree saturation under heavy load [25] and could become a key performance bottleneck.

Tree saturation occurs when multiple packets contend for a single resource (e.g., a link between nodes) creating a hotspot. Since only one packet can use the resource, other packets must wait. These waiting packets occupy buffers and thus delay other packets, even though they may be destined for completely different nodes and share only one link on their paths to their respective destinations. This process continues, waiting packets delay other packets producing a tree of waiting packets that fans out from the original hot-spot.

- M. Thottethodi is with the School of Electrical and Computer Engineering, Purdue University, 465 Northwestern Ave., West Lafayette, IN 479072035. E-mail:mithuna@purdue.edu.

- A.R. Lebeck is with the Department of Computer Science, Duke University, Box 90129, Levine Science Research Center, Research Dr., Durham, NC 27708.E-mail: alvy@cs.duke.edu.

- S.S. Mukherjee is with Intel Corporation, 334 South Street, Mail Stop: SHR1-T25, Shrewsbury, MA 01545. E-mail: shubu.mukherjee@intel.com.

Manuscript received 20 Mar. 2001; revised 10 Apr. 2002; accepted 6 Aug. 2003.

For information on obtaining reprints of this article, please send e-mail to: tpds@computer.org, and reference IEEECS Log Number 113826.
The performance degradation caused by network saturation can be severe, as illustrated in Fig. 1. Fig. 1 plots the delivered throughput (measured by simulation) in a 16-ary 2cube network with fully adaptive routing and deadlock recovery against various offered loads. The y-axis corresponds to delivered bandwidth (packets/node/cycle) while the $x$-axis shows the offered load in terms of packet injection rate (packets/node/cycle) of 16-flit packets. The two lines correspond to different communication patterns: randomly selecting a destination node (random) and using the node number with its bits inverted as the destination (complement).

Consider the curve for the random communication pattern in Fig. 1. We see that delivered throughput increases with offered load at lower loads, but at higher loads, the network eventually saturates and suffers significant performance degradation. Extending the discussion to the curve for the complement communication pattern, we can make two important observations. First, both communication patterns incur dramatic reductions in throughput when the network reaches saturation. The second observation is that the network saturates at different points for the different communication patterns.

One way to prevent network saturation is to use source throttling which prevents source node packet injection when congestion is detected. An oracle could achieve this by knowing exactly when congestion is imminent and throttling new packets just in time to maximize performance and prevent saturation. Unfortunately, doing this in a realistic implementation is a challenge because in real systems, nodes are typically aware of local information only (i.e., local buffer occupancy, local queue delays, whether the neighbouring nodes are prepared to accept packets/flits, etc.). It is difficult to detect imminent saturation by using purely local information. 


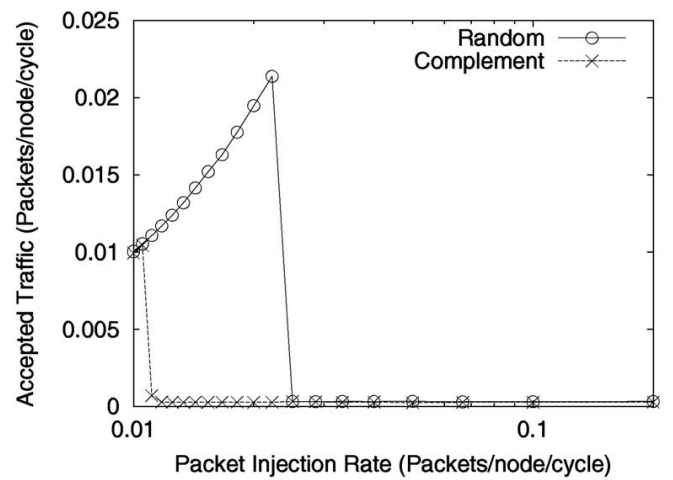

Fig. 1. Performance breakdown at network saturation, 16-ary 2-cube network, adaptive routing, and deadlock recovery.

This paper presents a global-knowledge based self-tuned source throttling mechanism for multiprocessor interconnection networks. Our solution is comprised of two key components: a technique to obtain global knowledge of network state and a self-tuning mechanism to automatically determine when network saturation occurs.

We use global knowledge of the number of full network buffers to estimate network congestion. Global information allows us to detect congestion earlier than alternative approaches that wait for network backpressure to create locally observable indicators of congestion (e.g., local buffer occupancy, timeouts). The global estimate is compared against a threshold to control packet injection. If the estimate is higher than the threshold, packet injection is stopped. When the estimate drops below the threshold, packet injection is resumed.

The second key aspect of our source throttling implementation is a self-tuning mechanism that monitors global network throughput and automatically determines the appropriate threshold value. This eliminates manual tuning and allows our scheme to adjust to variations in communication patterns.

We believe that our congestion control mechanism is generally applicable to a broad range of packet-switched, multiprocessor networks, including virtual cut-through [18] networks and wormhole networks [7], [6]. However, in this paper, we evaluate the technique in the context of wormhole switched, $k$-ary $n$-cube networks.

Our technique applies to all systems that use fine-grain messaging over the interconnection network and is agnostic on whether these messages are generated by hardware or software. For example, in multiprocessor systems with distributed memory and a shared address space [30], the hardware generates request and response messages on remote memory accesses. In contrast, software distributed shared memory systems such as Blizzard-S [28] and Shasta [27], use software generated fine-grain messaging over the interconnection networks of multicomputers (i.e., systems without hardware-supported, shared address spaces). In both cases, the interconnection network is expected to provide high-bandwidth, low latency communication at all offered loads.

Simulation results for a 16-ary 2-cube (256 node network) show that our congestion control technique prevents the severe performance degradation caused by network saturation. By limiting packet injection, our scheme sustains high throughput and low latency. Compared to an alternative approach that uses local estimates of congestion [2], our scheme is superior because global congestion estimation enables our technique to detect congestion in its early stages. We also show that a single static threshold cannot accommodate all communication patterns because a single threshold overthrottles some workloads and does not prevent saturation in other ones. In contrast, simulations reveal that our self-tuning technique automatically adapts to various communication patterns, including bursts of different patterns.

The remainder of this paper is organized as follows: Section 2 provides background information and discusses related work. Section 3 and Section 4 discuss the two key innovations of this paper. Section 3 presents our proposed global information gathering scheme. Section 4 describes our self-tuned congestion control scheme. Section 5 presents our experimental methodology and simulation results. Section 6 summarizes this paper.

\section{Background and Related Work}

High performance interconnection networks in tightly coupled multiprocessors can be achieved by using wormhole [7], [6] or cut-through switching [18], adaptive routing [14], and multiple virtual channels [4]. The Cray T3E [30] and SGI Origin [21] machines use a combination of these techniques for their interconnection networks. In these systems communication occurs by sending packets of information that are routed independently through the network. Each packet is composed of flits (flow control units) that are transferred between network nodes. ${ }^{1}$ Both wormhole routing and cut-through switching can suffer from network saturation. In wormhole switching, when a node receives the header flit (which typically contains the routing information), it immediately selects a route and forwards the flit to the next node. This can provide very low latency compared to store-and-forward routing where the entire packet is received by a node before forwarding it. However, when a packet blocks in a wormhole network, its flits occupy buffer space across several network nodes, and can exacerbate tree saturation. In contrast, routers using cutthrough switching buffer blocked packets in the router itself. Nevertheless, even cut-through switching can suffer from tree saturation when the router buffers fill up.

Adaptive routing dynamically chooses from multiple potential routes based on current local network state. This can help alleviate the effects of tree saturation experienced by deterministic routing algorithms under heavy load, and thus provide higher performance. Unfortunately, fully adaptive routing can cause potential deadlock cycles, which can exacerbate network saturation. While adaptive routing helps alleviate light to moderate congestion, in this paper we focus on source-throttling as a congestion control technique to prevent network saturation.

Virtual channels allow multiple packets to share a single physical link, thus reducing the effects of tree saturation, and can be used to eliminate deadlocks. Deadlock avoidance schemes work by ensuring that some virtual channels

1. For ease of exposition, we assume that each network node contains a processor, memory and a network router. 
are never in cyclic dependencies [9], [5]. In particular, we consider a scheme that reserves a small set of virtual channels for deadlock-free routing [9], while the remaining virtual channels use fully adaptive routing. This technique guarantees forward progress, since packets routed over the special channels will never deadlock, and eventually free up resources for the fully adaptive channels.

Deadlock recovery [20] is an alternative to deadlock avoidance that can potentially achieve higher performance. Deadlock recovery uses full adaptive routing on all virtual channels, detects when deadlocks occur (typically via timeouts), then recovers by routing packets on a deadlock free path which uses a central per-node buffer. This approach can also be imagined as containing two virtual networks: one can suffer deadlock and the other is guaranteed to be deadlock-free. The main difference is that this approach uses a per-node buffer whereas the deadlock avoidance scheme requires buffers per physical channel.

In either deadlock avoidance or recovery, the frequency of deadlocks in the adaptive channels increases dramatically when the network reaches saturation [33]. When this occurs, packets are delivered over the relatively limited escape bandwidth available on the deadlock-free paths. This causes a sudden, severe drop in throughput and corresponding increase in packet latency. Therefore, it is crucial to avoid network saturation in these systems.

To keep the network below saturation and avoid the resulting performance degradation, it is necessary to implement a congestion control mechanism. This mechanism should be self-tuning, thus eliminating the need for a system designer, administrator, or application programmer to tune various parameters and allowing the system to adapt to changes in communication patterns, load levels, and network topologies. Such a self-tuned system may require timely information about the global network state to correctly tune the congestion control algorithm.

The remainder of this section examines prior congestion control mechanisms for tightly coupled multiprocessors in the context of these desirable properties. We also examine congestion control mechanisms used in LANs/WANs and discuss the applicability of those techniques to tightly coupled multiprocessors.

\subsection{Related Work}

Most previous work on congestion control for multiprocessor networks relies on estimating network congestion independently at each node and limiting packet injection when the network is predicted to be near saturation. This reduces the problem to finding a local heuristic at each node to estimate network congestion. Lopez et al. [22], [23] use the number of busy output virtual channels in a node to estimate congestion. Baydal et al. [2] propose an approach that counts a subset (free and useful) of virtual channel buffers to decide whether to throttle or not. Because the above schemes rely on local symptoms of congestion, they lack knowledge about the global network state, and are unable to take corrective action in a timely manner. This reduces their effectiveness under different network load levels and communication patterns.

Smai and Thorelli describe a form of global congestion control [31]. A node that detects congestion (based on timeouts) signals all the other nodes in the network to also limit packet injection. This approach requires tuning the appropriate time-outs, and when the timeouts are tuned for robustness at higher loads, there is a performance penalty for light loads. The technique proposed by Kim et al. [19] allows the sender to kill any packet that has experienced more delays than a threshold. This approach pads shorter packets to ensure that the sender can kill a packet at any time before its first flit reaches the destination. This can cause larger overheads when short messages are sent to distant nodes. Additionally, both these schemes have been evaluated with uniform random traffic only and use a single static threshold. They do not discuss the need for selftuning, which arises when we consider diverse communication patterns.

Scott and Sohi describe the use of explicit feedback to inform nodes when tree-saturation is imminent in multistage interconnection networks [29]. This approach, which also requires tuning of thresholds, is not applicable to the class of networks we consider (direct networks, $k$-ary $n$-cubes).

The above techniques for congestion control in multiprocessor networks all attempt to prevent network saturation at heavy loads. Unfortunately, these techniques either require tuning, lack necessary information about a network's global state to take preventive actions in a timely fashion, or do not provide high performance under all traffic patterns and offered load levels.

Flit-reservation flow control is an alternative flow control technique which improves the network utilization at which saturation occurs [24]. It uses control flits to schedule bandwidth and buffers ahead of the arrival of data-flits. This prescheduling results in better reuse of buffers than waiting for feedback from neighboring nodes to free up buffers. Basak and Panda demonstrate that consumption channels can be a bottleneck that can exacerbate tree saturation. They show that saturation bandwidth can be increased by having an appropriate number of consumption channels [1]. Both these approaches (of improving network utilization at which saturation occurs) are orthogonal to our saturation prevention technique.

LANs (Local Area Networks) and WANs (Wide Area Networks) use self-tuned congestion control techniques. Various flavors of self-tuning, end-to-end congestion avoidance and control techniques have been used in the TCP protocol [16], [3]. TCP's congestion control mechanism uses time-outs and dropped/unacknowledged packets to locally estimate global congestion and throughput. If congestion is detected, the size of the sliding window, which controls the number of unacknowledged packets that can be in flight, is reduced. Floyd and Jacobson [12] proposed a scheme where TCP packets are dropped when a router feels that congestion is imminent. Dropped packets give an early indication to end hosts to take corrective action and scale back offered load. Floyd [11] also proposed a modification of TCP where "choke packets" are explicitly sent to other hosts. Ramakrishnan and Jain describe a similar mechanism for DECbit to explicitly notify congestion whereby gateways set the ECN (Explicit Congestion Notification) bit depending on average queue size [26].

Congestion control in ATM [17] uses explicit packets called Resource Management (RM) cells to propagate congestion information. Switches along the packet path modify bits in the RM cells to indicate the highest data-rate they can handle. The end-hosts are limited to using the maximum data-rate indicated by the switches to not overwhelm the network and/or switches.

The above congestion control mechanisms for LANs and WANs are not directly applicable in multiprocessor networks. LANs and WANs can drop packets because higher 
network layers will retransmit dropped packets for reliable communication. The dropped packets serve as implicit hints of network congestion. However, multiprocessor networks are typically expected to guarantee reliable communication. Thus, additional complexity would have to be built-in to store and retransmit dropped packets. The alternative idea of propagating congestion information explicitly can be used.

The challenge is in determining the appropriate set of mechanisms and policies required to provide a self-tuned congestion control implementation for preventing saturation in multiprocessor networks. In this paper, we present our solution for regular interconnection networks with adaptive routing, wormhole switching, and either deadlock recovery or deadlock avoidance.

Our solution is based on two key innovations that collectively overcome the limitations of previous congestion control techniques.

First, we use a global knowledge-based congestion estimation that enables a more timely estimate of network congestion. In Section 3, we describe how each node sends out its local state and receives aggregated global state. The second component is a self-tuning mechanism that automatically determines when saturation occurs allowing us to throttle packet injection. We describe, in Section 4, how each node runs a local algorithm to implement our self-tuning mechanism by interpreting the gathered global data and taking suitable action to prevent saturation.

\section{Global Congestion Estimation}

Any congestion control implementation requires a timely way to detect network congestion. Till now, we have used the term "congestion" in a qualitative way to imply network conditions wherein the network achieves poor throughput and packets suffer from high latencies. We need a way to quantitatively measure congestion. This measure can then be compared to a threshold to determine if packets should be throttled or not. This is typically done by measuring the "symptoms" that manifest themselves when the network is congested (such as increased routing failures, increased buffer occupancy and increased queue delays). Previous techniques estimate network congestion using a locally observable quantity (e.g., local virtual buffer occupancy, packet timeouts, etc.). While these estimates are correlated to network congestion, we claim that waiting for local symptoms of network congestion is less useful primarily because, by that time, the network is already overloaded.

Consider the case when network congestion develops at some distance from a given node. Schemes that use local heuristics to estimate congestion rely on back-pressure to propagate symptoms of congestion to the node (e.g., filling up of buffers, increase in queue delays, etc.). The node takes no corrective action until congestion symptoms are observed locally.

It is possible to detect network congestion in its early stages by taking global conditions into account. To achieve this, we use the fraction of full virtual channel buffers of all nodes in the network as our metric to estimate network congestion. This ensures that remote congestion is accounted for early enough to take corrective action. However, there are additional costs, both hardware and latency, to propagate the global information.
Our scheme counts full buffers to estimate congestion but does not take the distribution of these full buffers among the nodes into account. At first glance, this appears to be a serious limitation because our scheme is unable to distinguish between a case with localized congestion (i.e., a large fraction of full buffers are in relatively few nodes in the network) and a benign case (in which the same number of full buffers are distributed more or less evenly among all the nodes of the network). But the adaptivity of our selftuning mechanism reduces the impact of this problem by setting the threshold differently in the two cases. Our mechanism will set a higher threshold for the benign case than for the case with localized congestion. (Note, the threshold will be different for the two cases, but for a given case, all nodes in the network will arrive at the same threshold since they are using the same global information. As such, all nodes throttle new packets when the buffer occupancy exceeds the threshold.)

In the next section, we show how global information can be gathered with reasonable cost and used to achieve a robust, self-tuned congestion control implementation.

\subsection{Implementing Global Information Gather}

Our technique requires that every node in the network be aware of the aggregate number of full buffers and throughput for the entire network. (We explain the relationship between full buffers, offered load and delivered bandwidth in Section 4.) There are a variety of ways to implement this allto-all communication. In this section we discuss two alternatives: metapackets, and a dedicated side-band.

One approach is to send out special metapackets containing the congestion and throughput information. The required all-to-all communication can be guaranteed by this approach. However, guaranteeing delay bounds may involve additional complexity. Metapackets flooding the network will also consume some of the bandwidth and may add to the congestion. Adding a high-priority virtual channel reserved for these metapackets is one way to address these concerns. Our experimental results (in Section 5.3) demonstrate that using metapackets for global information distribution causes our congestion control scheme to fail because of two reasons. The additional congestion caused by the metapacket distribution causes some degradation in achieved throughput. Further, this information does not arrive early enough to be useful. Using the stale information to drive our throttling mechanism causes further degradation in throughput.

For this paper, we use an exclusive side-band reserved for communicating the congestion and throughput information. This is a costlier implementation in terms of additional hardware and complexity. However, it is easy to guarantee delay bounds on all-to-all communication and it does not affect performance of the main data network. While the extra bandwidth available on the side-band could be used for general packet routing, it will only postpone network saturation for a short-time, and not provide a complete solution like our congestion control scheme. Some other networks also use a sideband to transmit meta information to better manage the network. The SPIDER router [13] uses 32 bits of control overhead per 128 bit data-packet, out of which a sideband channel of 8 bits is used to tag virtual channels and to implement credit-based flow-control. The 

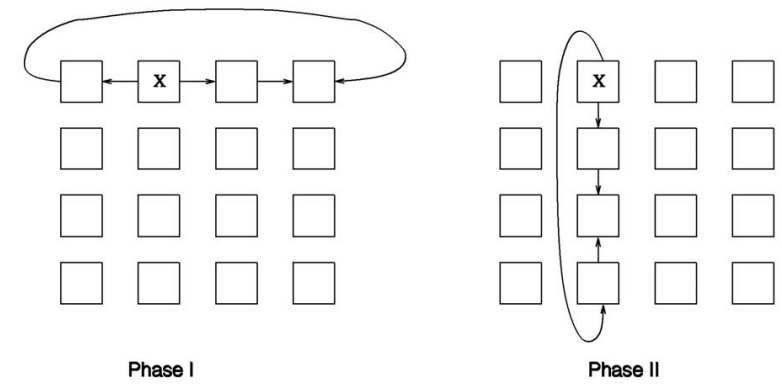

Fig. 2. Dimension-wise global aggregation.

HiPPI-6400 network standard [15] similarly uses 64 bits of control overhead per 256 bit micropackets.

The side-band incurs a neighbor-to-neighbor communication delay of $h$ cycles. We use a dimension-wise aggregation scheme (Fig. 2). Every node communicates its number of full buffers and throughput in both directions (Phase I in Fig. 2) along the lowest dimension of the network. (Note, Fig. 2 shows the communication of one node in the network. Every node is simultaneously participating in such an information exchange.) Each node that receives such information computes the aggregate and has the aggregate information for all its neighbors along the zeroth dimension at the end of $k / 2$ hops or $(k / 2) * h$ cycles. The nodes then communicate the aggregates to neighbors along the next higher dimension (Phase II in Fig. 2).

Continuing this procedure along every dimension, global aggregation in a full-duplex, $k$-ary $n$-cube network completes in $(k / 2) * h * n$ cycles. Assuming $h=2$, for our network configuration $(n=2, k=16)$ it takes 32 cycles. We refer to the time for such an all-to-all communication as the gather-duration $(g)$.

The mechanism described above provides $g$-cycle delayed snapshots of the network congestion every $g$ cycles. Our congestion control policy requires us to compare, in every cycle, the current estimated congestion to the threshold. If we're currently at time $t$ and we have observed previous network snapshots at $S_{2}, S_{1}, S_{0}$, etc., we must estimate the network congestion at time $t$ based on the previous snap-shots of global network congestion.

The simplest solution is to use the state observed in the immediately previous network snapshot until the next snapshot becomes available. We use a slightly more sophisticated method to estimate network congestion that computes a linear extrapolation based on the previous two network-snapshots. In general, any prediction mechanism based on previously observed network states can be used to predict network congestion. We leave evaluation of alternative prediction techniques for future work. On average, we found the linear extrapolation technique yields an improvement in throughput of 3 percent for the deadlock avoidance configuration and 5 percent for the deadlock recovery configuration.

To communicate congestion information, nodes exchange full buffer counts. The number of bits needed to represent this information depends on the number of buffers in the network. We specify the network configuration we use and the number of bits needed to represent congestion information for that configuration in Section 5.

In summary, a global aggregate of buffer occupancy provides an early estimate of network congestion. This

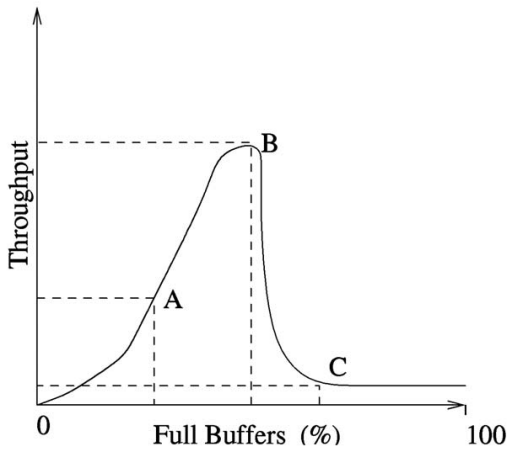

Fig. 3. Throughput versus full buffers.

estimate is compared against a threshold to determine if packet injection should stop or resume. Obtaining information on the global aggregate buffer occupancy of the network is only a part of the solution. To translate this congestion estimate to good congestion control, we have to properly choose the threshold. Our self-tuning mechanism, described in the next section, dynamically tunes the threshold to the appropriate values.

\section{A Self-Tuning Mechanism}

Proper threshold selection is a crucial component of our congestion control implementation. Inappropriate threshold values can produce unstable behavior at high loads or unnecessarily limit performance for light loads. Furthermore, there is no single threshold that works well for all communication patterns. This section presents a technique to automatically determine the proper threshold value.

The goal of our self-tuning mechanism is to maximize delivered throughput without dramatic increases in packet latency. Therefore, we can view our task as an optimization problem with delivered bandwidth as an objective function dependent on the number of full virtual buffers. Intuitively, we know that buffer occupancy (and achieved throughput) will be low at low loads because of the small number of packets in flight. Both buffer occupancy and achieved throughput will increase at higher loads as the number of packets in flight increases. However, beyond saturation load, the number of full buffers keeps increasing due to blocked packets but there is a drop in achieved throughput. Fig. 3 illustrates this intuition about the relationship between offered load, full buffers and delivered bandwidth.

Our self-tuning technique attempts to find the number of full virtual buffers (i.e., the threshold value) that maximizes delivered throughput (B in Fig. 3). To achieve this, we use a hill-climbing algorithm including a technique to avoid local maxima. We can obtain a measure of global network throughput (the objective function) in a manner similar to the way we obtain the global count of full virtual buffers (see Section 3). Nodes exchange the number of flits delivered in the past $g$ cycles to measure throughput. If we consider the maximum possible delivered bandwidth of 1 flit/node/ cycle, the count will not exceed $g *$ NodeCount.

\subsection{Hill Climbing}

To automatically tune the threshold, we begin with an initial value based on network parameters (e.g., 1 percent of all buffers). We use the intuition about the relationship 
TABLE 1

Tuning Decision Table

\begin{tabular}{|c|c|c|}
\hline Drop in Bandwidth $>25 \% ?$ & \multicolumn{2}{|c|}{ Currently Throttling? } \\
\cline { 2 - 3 } & Yes & No \\
\hline Yes & Decrement & Decrement \\
\hline No & Increment & No Change \\
\hline
\end{tabular}

between the number of full buffers and delivered bandwidth (see Fig. 3) to specify a tuning decision table that indicates when the threshold value must be increased or decreased. Too low a value (A or lower in Fig. 3) prevents us from reaching the peak by over throttling packet injection. In contrast, too high a value pushes us beyond the peak (C or higher in Fig. 3), causing saturation just like a network without any congestion control.

Table 1 illustrates the tuning decision table. The two dimensions in the table correspond to observed network throughput and whether or not the network is currently throttled. We make a tuning decision once every tuning period. We say that there is a drop in bandwidth only if the throughput in a tuning period drops to less than a specified fraction (we use 75 percent $^{2}$ ) of the throughput in the previous tuning period. We leave more complex schemes that adaptively modify the frequency of tuning according to network conditions as future work.

The tuning period is an exact multiple of the gatherduration. If the tuning period is very large, there is likely to be slow and inefficient tuning leading to network underutilization or network saturation. If it is too small, shortlived crests and troughs in throughput could alter the estimate. However, in our experiments, we found that, for a reasonable range of values (32 cycles to 192 cycles) the performance did not alter significantly. In our experiments, we use a 96 cycle tuning period.

The tuning process proceeds as follows. If we observe a decrease in throughput (upper row in Table 1), then we always decrease the threshold value. The decreased throughput is a result of either network saturation or a decrease in offered load. If the cause is saturation, then we must reduce the threshold value to bring the network out of saturation. If the offered load has decreased, then it is still safe to reduce the threshold since the system is not overthrottling.

If the system is throttling and there is no drop in throughput, we optimistically increase the threshold. If there is no drop in throughput after the increase, our optimism was justified and the lower threshold value was overthrottling. If we exceed the saturation point because of the increase in threshold, the observed bandwidth decreases and we again reduce the threshold value. Finally, if throttling is not occurring and there is no decrease in throughput, we do not change the threshold value.

We use constant additive increments and decrements to update the threshold value. There could be other, more sophisticated, algorithms to tune the threshold that improves the tuning mechanism by reducing the time to reach the "sweet spot." We find constant additive tuning adequate for

2. Setting a very high fraction increases the likelihood of thrashing because of normal, minor fluctuations in throughput. A very low fraction makes the tuning mechanism insensitive to drops in bandwidth. We chose to use 75 percent fraction after a few experiments. effective self-tuning and do not explore other, more complicated methods in this paper. For a reasonable range of values, (1 percent to 4 percent of all buffers) performance is insensitive (within 4 percent) to variations in increment/ decrement values. There is marginally better performance when the decrement is higher than the increment. We use an increment of 1 percent of all buffers and a decrement of 4 percent of all buffers. For our 16-ary 2-cube network this corresponds to an increment of 30 and a decrement of 122.

Since we're only comparing throughput observed in each tuning period to the throughput observed in the previous tuning period, it is possible that if the bandwidth drop due to saturation happens gradually, we will not treat it as sufficient to trigger a decrease in threshold value. Thus, the network "creeps" into saturation and hits a local maximum. The hill climbing technique, as currently proposed fails to move back from this local maximum. Increasing the threshold beyond this keeps the network in saturation with no further drop in bandwidth. In the next section, we describe a method to scale back the threshold away from the local maximum.

\subsection{Avoiding Local Maxima}

To avoid settling at local maxima, we remember the conditions that existed when maximum throughput was achieved. To do this, we keep track of the maximum throughput $(\max )$ achieved during any single tuning period and remember the corresponding number of full buffers $\left(N_{\max }\right)$ and threshold $\left(T_{\max }\right)$.

If the throughput in any tune-period drops significantly below the maximum throughput, our technique tries to recreate the conditions that existed when maximum throughput was achieved. We do this by setting the threshold to $\min \left(T_{\max }, N_{\max }\right)$. If $N_{\max }$ is higher than $T_{\max }$, it means that the network was throttling with a threshold value of $T_{\max }$ when it achieved the maximum observed throughput. In this case, we set the threshold to $T_{\max }$ so that the network can throttle new packets and drain existing packets till it reaches the desired load level. If, on the other hand, $N_{\max }$ is smaller than $T_{\max }$, then setting the threshold to $N_{\max }$ is a better choice because it is possible that $T_{\max }$ is not low enough to prevent saturation. This guarantees that we're not stuck at a local maximum after the network saturates.

It is possible that the threshold value which sustains high throughput for one communication pattern is not low enough to prevent saturation for another communication pattern. Our congestion control mechanism detects and adapts the threshold to such changes. If we find that we reset the threshold to $\min \left(T_{\max }, N_{\max }\right)$ for $r$ consecutive tuning-periods, this means that even the $\min \left(T_{\max }, N_{\max }\right)$ value is too high to prevent saturation, and we must recompute $\max$ value. In this case, we reset $\max$ to zero and restart the maximum-locating process. This ensures that our threshold adapts to changing communication patterns. We use $r=5$ in our experiments.

\subsection{Summary}

The above discussion provides a general overview of a technique we believe can provide a robust, self-tuned congestion control. Our scheme gathers full-buffer counts and throughput measurements every 32 cycles. The fullbuffer counts are used to estimate current congestion using linear extrapolation. This estimate is compared to a threshold 
TABLE 2

Destination Nodes for Source Node $N_{154}$ (Bit Coordinates (10011010)) in a 16-ary 2-cube

\begin{tabular}{|c|c|c|c|}
\hline Traffic Pattern & $\begin{array}{c}\text { Destination } \\
\text { (Bit transformation) }\end{array}$ & $\begin{array}{c}\text { Destination } \\
\text { (Bit coordinates) }\end{array}$ & $\begin{array}{c}\text { Destination } \\
\text { (Node number) }\end{array}$ \\
\hline Random & Any & Any & Any \\
Perfect Shuffle & $a_{n-2}, \ldots, a_{1}, a_{0}, a_{n-1}$ & 00110101 & $N_{53}$ \\
Complement & $a_{n-1}, a_{n-2}, \ldots, a_{1}, a_{0}$ & 01100101 & $N_{101}$ \\
Bit Reversal & $a_{0}, a_{1}, \ldots, a_{n-2}, a_{n-1}$ & 01011001 & $N_{89}$ \\
\hline
\end{tabular}

to decide whether we throttle new packets or not. We use a hill climbing algorithm to update our threshold every 96 cycles in increments and decrements of 1 percent and 4 percent of total buffer count, respectively. Our hill climbing algorithm, when used alone, is susceptible to settling on local maxima after network saturation. Our scheme includes a mechanism to prevent this from happening by remembering maximum ( $\max )$ observed throughput. Finally, we recompute the maximum $(\max )$ if we reset the threshold for $r=5$ consecutive tuning periods.

On a high level, our scheme is somewhat analogous to TCP's self-tuning congestion control. Both have an idea of what the network performance should be: expected roundtrip time (RTT) in the case of TCP and max throughput in our case. Both schemes allow offered load to incrementally increase as long as network performance is not penalized. The sliding window size increases as long as no packets are dropped in the case of TCP and threshold increases as long as there is no decrease in throughput in our case. Both techniques take corrective action if network performance suffers. TCP reduces its window size and our scheme either decrements the threshold or resets it to $\min \left(N_{\max }, T_{\max }\right)$. One difference is that while TCP does a multiplicative decrease, our hill-climbing scheme does an additive decrease on the threshold. We observed the multiplicative backoff to be too conservative (i.e., over throttling) and that additive decrease (with bigger decrements than increments) was as robust without over-throttling. Section 5.2.3 shows experimental results that show how the threshold is tuned in our scheme. Finally, both schemes periodically refresh their estimate of network performance. TCP recomputes expected round-trip-time if packets are dropped, whereas our scheme recomputes $\max , N_{\max }$ and $T_{\max }$ if $\max$ is stale, i.e., if there are $r$ consecutive corrective actions.

\section{Evaluation}

This section uses simulation to evaluate our congestion control technique. We begin by describing our evaluation methodology. This is followed by our simulation results.

\subsection{Methodology}

To evaluate our congestion control scheme, we use the flexsim [32] simulator. We simulate a 16-ary 2-cube network (256 nodes) with full duplex physical links. Each node has one injection channel (through which packets sent by that node enter the network) and one delivery channel (through which packets sent to that node exit the network). We use three virtual channels per physical channel and edge-buffers (buffers associated with virtual channels) which can hold eight flits.
The router's arbiter is a central resource which only one packet can use at a time and there's a one cycle routing delay per packet header. Packets obtain control of the router's arbiter on a demand-slotted round-robin distribution. This is not a bottleneck because routing occurs only for the header flit of a 16-flit packet. The remaining flits simply stream behind the header flit along the same switch path. It takes one cycle per flit to traverse the cross-bar switch and one cycle per flit to traverse a physical link.

We evaluate our congestion control mechanism with both deadlock avoidance and deadlock recovery mechanisms. Deadlock avoidance uses the method proposed by Duato [9] with one escape virtual channel using oblivious dimension-order routing. We use the Disha [20] progressive deadlock recovery scheme with a time-out of 8 cycles.

All simulations execute for 60,000 cycles. However, we ignore the first 10,000 cycles to eliminate warm-up transients. Most results are presented in two parts: normalized delivered throughput (accepted flits/node/cycle) and average packet latency versus offered load in terms of packet injection rate.

The default load consists of each node generating 16 flit packets at the same fixed rate. We consider four different communication patterns, uniform random, bit-reversal, perfectshuffle, and complement. These communication patterns differ in the way a destination node is chosen for a given source node with bit coordinates $\left(a_{n-1}, a_{n-2}, \ldots, a_{1}, a_{0}\right)$. The bit coordinates for the destination nodes are $\left(a_{n-2}, a_{n-3} \ldots, a_{0}, a_{n-1}\right)$ for perfect shuffle, $\overline{\left(a_{n-1}, a_{n-2}, \ldots, a_{1}, a_{0}\right)}$ for complement and $\left(a_{0}, a_{1}, \ldots, a_{n-2}, a_{n-1}\right)$ for bit-reversal. Table 2 shows the destination selection for all the considered communication patterns for an example. If we assume that the nodes are canonically numbered from $N_{0}$ to $N_{255}$, Table 2 shows how destination nodes are selected for $N_{154}$.

In this paper, we present results for steady loads with uniform random communication pattern. We also present results for a bursty load with the various communication patterns. Results for steady loads with bit-reversal, perfect shuffle, and complement communication patterns are shown in Appendix A.

We use synthetic workloads instead of full-blown multiprocessor workloads, for three reasons: First, our simulation environment cannot handle full-blown multiprocessor workloads. Second, our packet generation frequency corresponds to realistic miss rates in databases and scientific applications, which gives us confidence in our results. Third, our synthetic workloads nicely demonstrate the problem of network saturation and avoids interactions with application-specific features.

For our network (with 3,072 buffers), 12 bits are enough to count all buffers in the network. Our configuration needs 


\section{Deadlock Recovery}

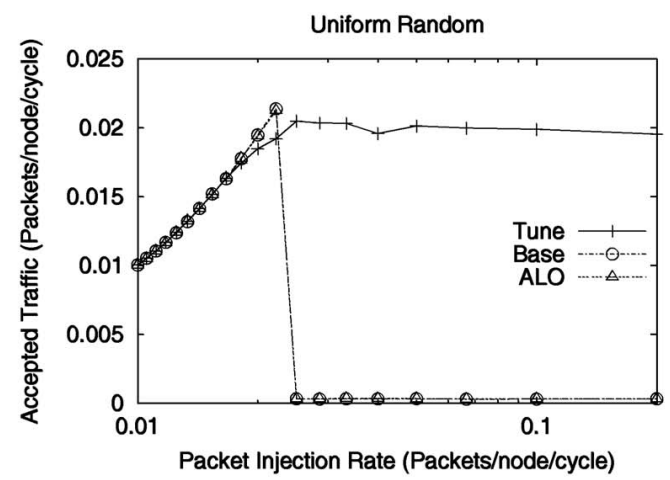

(a)

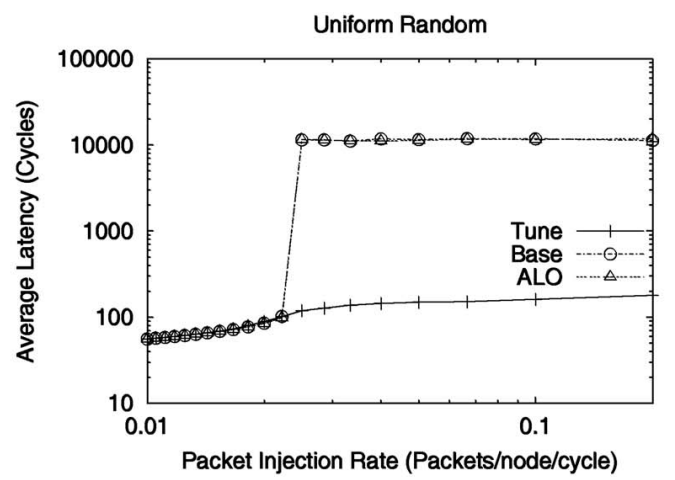

(b)

\section{Deadlock Avoidance}

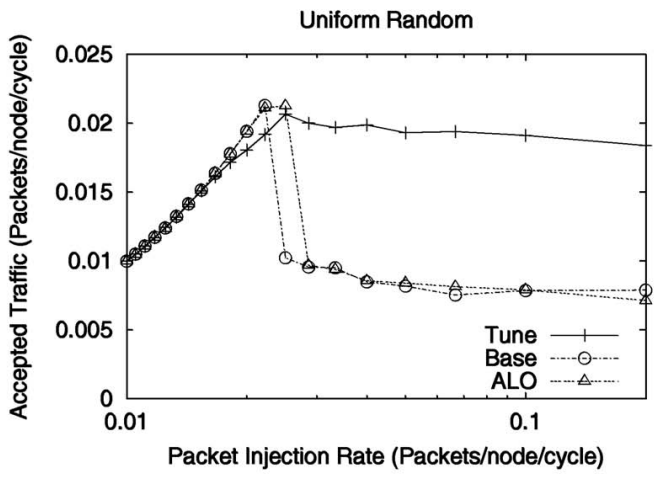

(c)

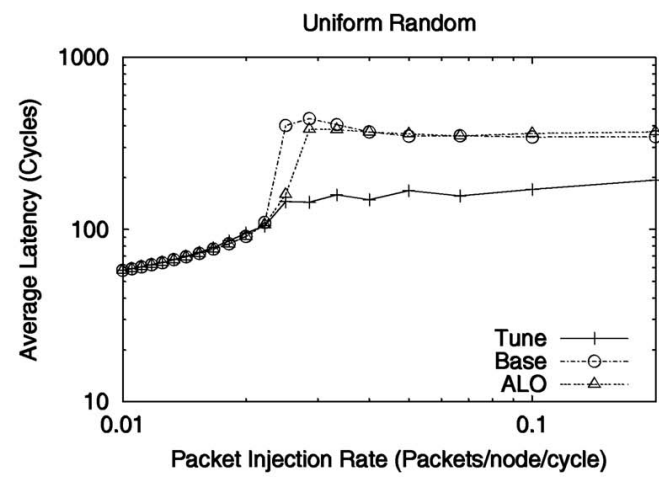

(d)

Fig. 4. Overall performance with random traffic. (a) and (c) Delivered throughput versus offered load. (b) and (d) Average latency versus offered load.

13 bits to represent the maximum possible aggregate throughput (one flit/cycle/node or a total of $g *$ NodeCount $*$ MaxTraffic $=32 * 256 * 1=8192$ flits). Thus, we need a total of 25 bits for the sideband signals. However, in Section 5.2.2, we show that we can send these 25 bits using 9-bit sideband channels with very little performance degradation.

For comparison, we also simulate the At-Least-One $(A L O)$ [2] congestion control scheme which has been shown to be better than other previous schemes such as [22], [23]. $A L O$ estimates global network congestion locally at each node. If at least one virtual channel is free on every $u s e f u l^{3}$ physical channel or if at least one useful physical channel has all its virtual channels free, then packet injection is allowed. Otherwise, new packets are throttled.

\subsection{Simulation Results}

The primary conclusions from our simulations are:

- Our technique provides high performance consistently across different communication patterns and offered load levels.

- Our technique outperforms an alternative congestion control technique that uses local estimates of congestion.

3. Useful is an output channel that can be used without violating the minimal-routing constraint.
- Our self-tuning technique adapts the threshold dynamically to varying workloads and to bursty traffic.

The remainder of this section elaborates on each of these items.

\subsubsection{Overall Performance}

We begin by examining the performance of a complete implementation, as described in Sections 3 and 4. Fig. 4 shows the bandwidth and latency for a uniform-random traffic pattern for both deadlock recovery (Figs. $4 \mathrm{a}$ and $4 \mathrm{~b}$ ) and deadlock avoidance (Figs. $4 \mathrm{c}$ and $4 \mathrm{~d}$ ). Note the logarithmic scale used on the y-axis for the latency graphs (Figs. $4 \mathrm{~b}$ and $4 \mathrm{~d}$ ).

The curve for the base case illustrates the network saturation problem. As load increases, the network throughput increases to a certain extent. However, at saturation, there is a sudden drop in throughput since only the escape channels are available to drain deadlocks. The configuration with deadlock recovery has lower bandwidth at saturation because Disha deadlock recovery requires that a packet obtain exclusive access to the deadlock-free path. In contrast deadlock avoidance schemes can break multiple deadlock cycles concurrently.

The results in Fig. 4 clearly show the key point that our congestion control technique (Tune) is stable at high loads. The $A L O$ congestion control scheme improves performance in the early stages of congestion for the deadlock avoidance case, but it does exhibit severe performance degradation 


\section{Deadlock Recovery}

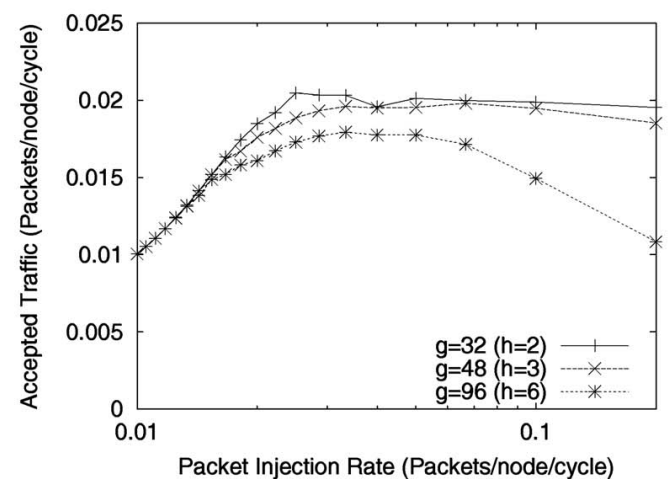

(a)

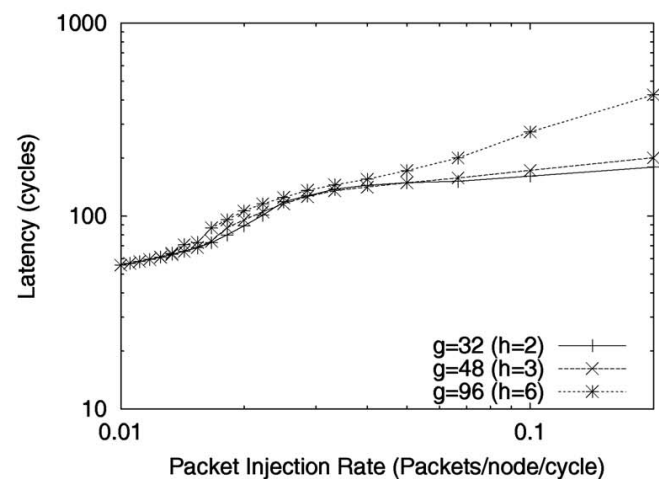

(b)

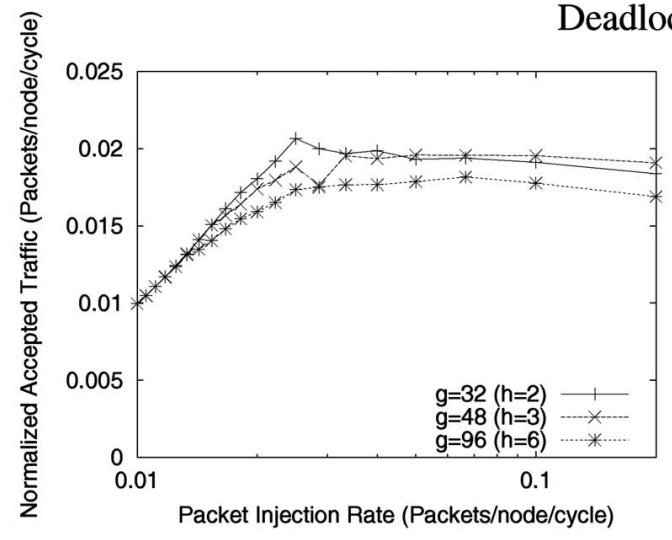

(c)

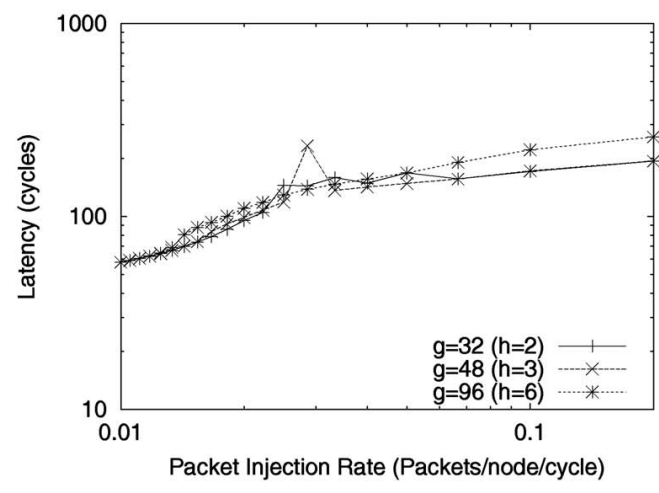

(d)

Fig. 5. Effect of global information gathering delays for deadlock recovery (a) delivered throughput versus offered load and (b) average latency versus offered load, and for deadlock avoidance (c) delivered throughput versus offered load and (d) average latency versus offered load.

eventually. Our scheme, however, maintains latency and throughput close to the peak values.

Fig. 13, 14, and 15 in Appendix A show similar results for other traffic patterns. One key difference that is seen is that our scheme seems to suffer higher latency than the base case (and ALO) at high loads. Taken in isolation, the latency data can be misleading. The lower average latency of the base (and ALO) scheme is for very few delivered packets (indicated by the low throughput) since most packets are still blocked in the network due to saturation. The few packets that are making forward progress on the deadlock free network might achieve lower latencies. But it is at the cost of reduced bandwidth. The throughput and latency graphs, when read in conjunction, show that our scheme has better performance at higher loads.

\subsubsection{Effect of Delay}

We consider the effect of varying the delay ( $h$, the time for one neighbor-to-neighbor communication on the side-band) to gather global information on overall performance. If $h=2$, that corresponds to a global-aggregation time $(g)$ of 32 cycles. For $h=3, g=48$, and for $h=6, g=96$. The tuning period remains 96 cycles.

Note that increasing the side-band communication delay can be viewed as equivalent to decreasing the width of the side-band by the same factor. For example, increasing the hop delay by a factor of three (from $h=2$ to $h=6$ ) can be alternately viewed as decreasing the side-band width by a factor of three (from 25 bits wide to 9 bits wide). The 25-bits of information can be sent out in three units taking 6 cycles, with transmission of each unit taking 2 cycles.

Intuitively, we know that stale information is less useful than fresh information. That is indeed the case if we use the stale information directly. However, with linear extrapolation, it is possible to estimate current information from stale information with some accuracy. In fact, for "noisy" curves,

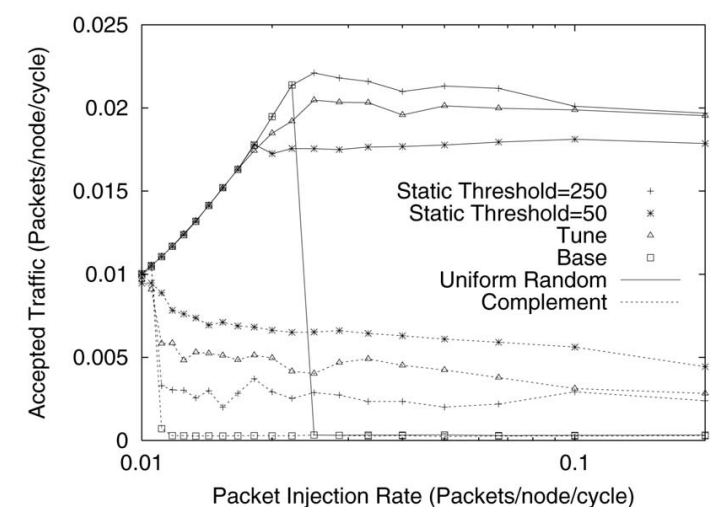

Fig. 6. Static threshold versus tuning. 


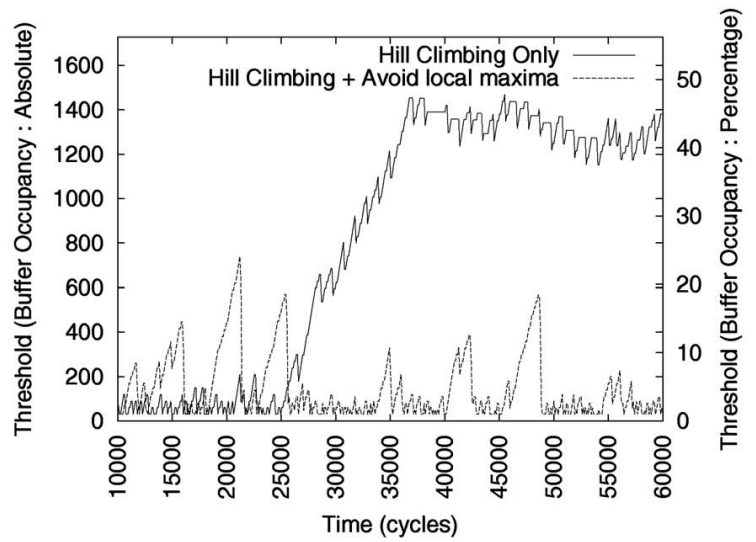

(a)

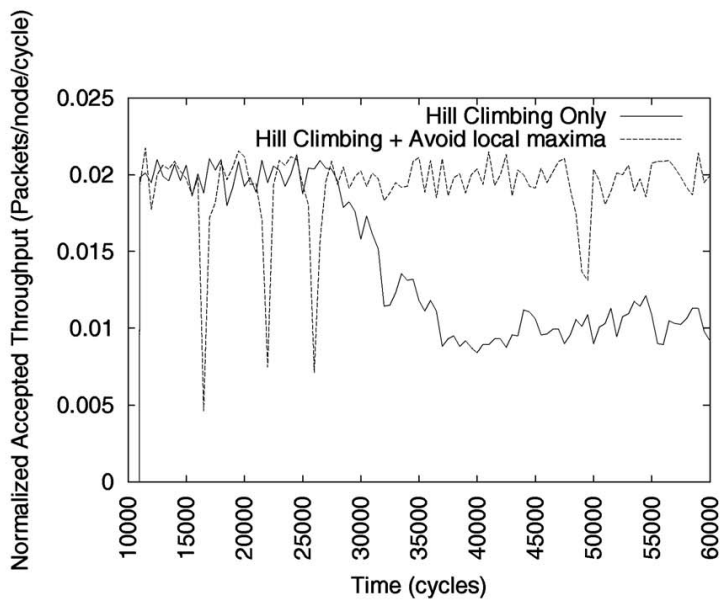

(b)

Fig. 7. Self-tuning operation: an example. (a) Threshold versus time. (b) Throughput versus time.

linear extrapolation may reflect the trends better than increased sampling.

Fig. 5 shows quantitatively the performance losses due to delays in gathering global information. We do see some points where our tuning scheme works better with a 48 cycle delay than with a 32 cycle delay. For the points in question, linear extrapolation gives an average increase in throughput of 10 percent over "previous snapshot" approach with a delay of 48 against an average increase in throughput of 4 percent with a delay of 32 . We also see that a delay of 96 cycles shows performance degradation due to very stale information.

\subsubsection{Self-Tuning}

In this section, we demonstrate two important aspects about our self-tuning technique. First, we show the importance of having a congestion control mechanism that adapts to the congestion characteristics of different communication patterns. This is followed by an examination of the hillclimbing algorithm and the scheme to avoid local maxima.

Recall from Fig. 1 that saturation occurs at different levels of offered load for random and complement communication patterns. These different levels of offered load correspond to different buffer occupancies in the network.

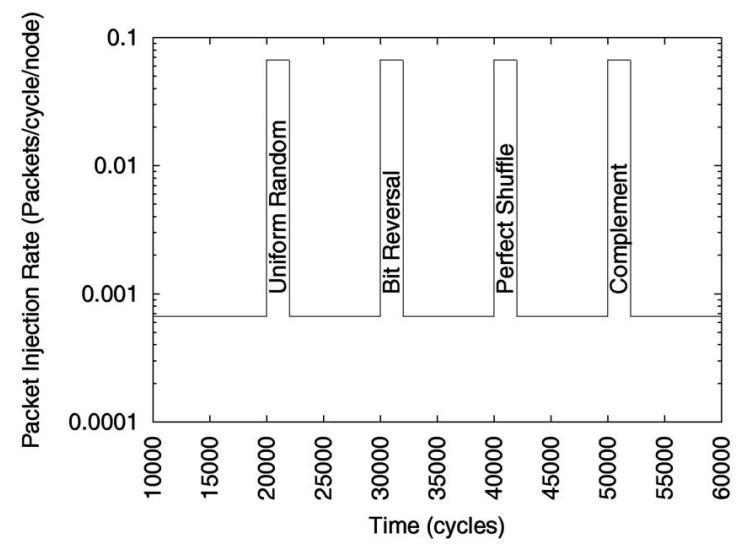

Fig. 8. Offered bursty load.
If saturation was occurring at the same buffer occupancies for different workloads, a well-chosen, single, static threshold could prevent network saturation. To show that this is not so, Fig. 6 compares the performance on the deadlock recovery network configuration of a congestion control mechanism with static thresholds to our scheme.

We consider uniform random (the four solid lines) and complement (the four dashed lines) communication patterns. We see that a static threshold of 250 (8 percent buffer occupancy) works very well for random traffic but the same threshold is unable to prevent saturation for the complement communication pattern. In contrast, a static threshold of 50 (1.6 percent buffer occupancy) works well for the complement communication pattern but over-throttles the random traffic. This indicates that the buffer occupancy at which the network saturates is not uniform across communication patterns. Therefore, it is necessary to have a self-tuning mechanism that adapts the threshold as communication patterns change.

To understand the behavior of our self-tuning technique, we analyze its operation for a specific configuration. As stated in Section 4, we use a gather-period ( $g$ ) of 32 cycles, a tuning period of 96 cycles, an increment of 1 percent of all virtual channel buffers and a decrement of 4 percent of all virtual channel buffers. The load is of uniform random distribution with a packet regeneration interval of 10 cycles and we use the deadlock avoidance configuration. With these parameters, Fig. 7a shows the tuning of the threshold over time for the duration of the simulation. Recall, the first 10,000 cycles are ignored to eliminate start-up transients. Fig. $7 \mathrm{~b}$ shows the throughput achieved over the same interval.

The hill climbing mechanism tries to increase the threshold as long as there is no decrease in bandwidth and tries to scale back when bandwidth decreases. But it can settle down at a local maximum when the decrease in bandwidth happens gradually. When this occurs, the network "creeps" into saturation and throughput falls.

Without a mechanism to avoid local maxima, the hill climbing algorithm can settle on a local maximum corresponding to deep saturation. The solid line in Fig. 7 shows this behavior. A gradual drop in throughput begins 


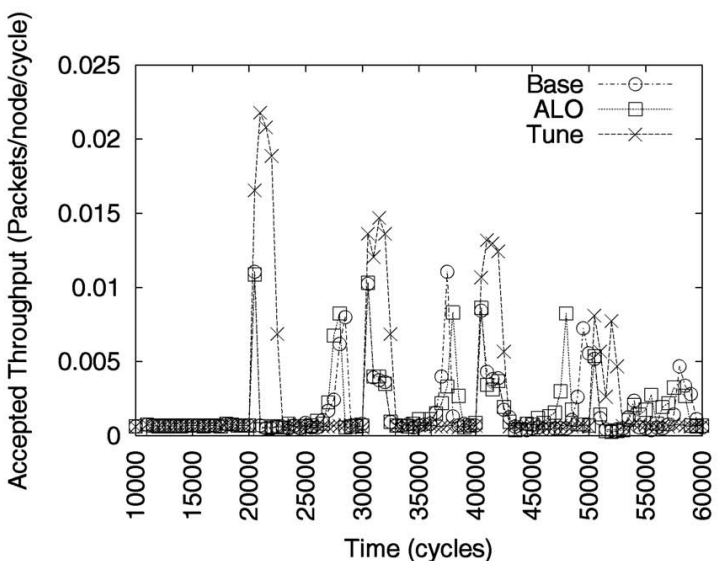

(a)

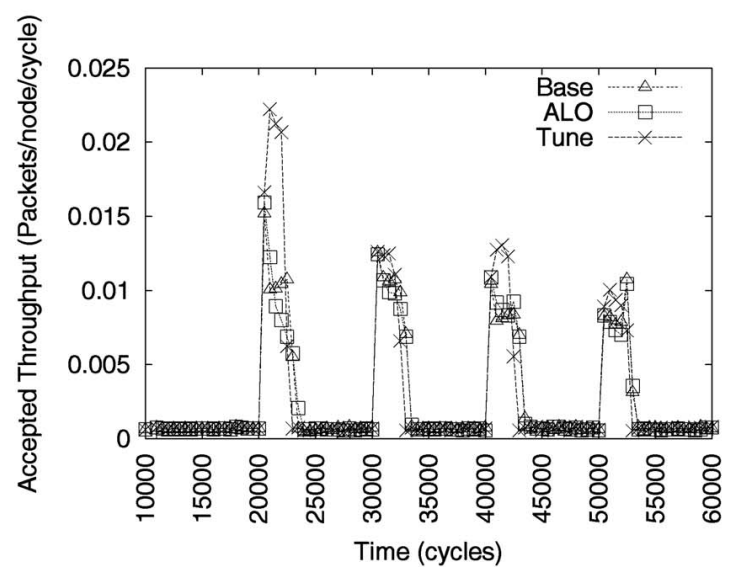

(b)

Fig. 9. Performance with bursty load: Delivered throughput with (a) deadlock recovery and with (b) deadlock avoidance.

at approximately 26,000 cycles. Recall that we decrement the threshold only when there is a throughput drop of 25 percent or more in any tuning period. We see, in Fig. 7a, that although there are many decrements, the gradual nature of the decrease in throughput results in an overall rise in the threshold, eventually saturating the network.

The dashed line in Fig. 7 shows the behavior of our scheme to avoid local maxima. The sharp dip in the threshold value (specifically the one at approximately 26,000 cycles) illustrates the corrective action taken when the network "creeps" toward saturation. As a result, we avoid saturation and sustain higher throughput.

\subsubsection{Bursty Traffic}

To confirm that our self-tuning mechanism works well under varying load, we use a bursty load created by alternating low loads and high loads. In addition, we also change the communication pattern for each high load burst. The offered bursty load is shown in Fig. 8. In the low load phase, the communication pattern is uniform random and every node tries to generate one packet every 1,500 cycle period (corresponding to a packet injection rate of 0.00067 packets / node/cycle). In the high load phase, every node tries to generate a packet every 15 cycles (corresponding to a packet injection rate of 0.067 packets / node/cycle). The communication pattern in each of these high load bursts is different and is indicated in Fig. 8.

Fig. 9a and Fig. 9b show the delivered throughput with bursty load for the deadlock recovery and the deadlock avoidance configurations, respectively. With deadlock recovery, the average packet latency for Base, $A L O$, and Tune configurations are 2,838 cycles, 2,571 cycles, and 161 cycles, respectively. With deadlock avoidance, the average packet latency for Base, $A L O$, and Tune configurations are 520 cycles, 509 cycles, and 163 cycles, respectively. In the high-load phase, our congestion control consistently delivers sustained throughput and predictable latencies. The $A L O$ scheme and the base scheme initially ramp up the throughput but throughput collapses soon thereafter due to network saturation.

The deadlock recovery results exhibit an interesting phenomenon in the Base and $A L O$ schemes (Fig. 9a). There are small bursts in throughput long after the offered load is reduced. This is because the network absorbs the heavy offered load but goes into deep saturation with many deadlock cycles. We observe this happening approximately between 20,000 and 21,000 cycles in Fig. 9a. There is a period when network packets are draining through the limited escape bandwidth available (approximately between 21,000 and 27,000 cycles). It is only when the deadlock cycles break that full adaptive routing begins again. The network then drains quickly showing the spurt in throughput (approximately between 27,000 and 29,000 cycles).

\subsection{Distributing Information Using Metapackets}

In this section, we evaluate an alternative mechanism to distribute global information. Buffer occupancy and throughput information are distributed in special metapackets over the same physical links as regular data-packets but they use an exclusive, additional virtual channel. The additional virtual channel for metapackets also has higher priority than other virtual channels to ensure delay bounds on information distribution.

Our experiments demonstrate that our congestion control scheme is not effective in preventing saturation when using metapackets as the information distribution mechanism. This is partly because of the contention for physical links caused by metapackets and partly because of the larger gather times resulting in stale information. In the rest of the section, we first describe our aggregation technique using metapackets and then present experimental results using 2-flit metapackets.

\subsubsection{Dimension-Wise Aggregation Using Metapackets}

As in the case of side-band, we aggregate quantities along lower dimensions before proceeding along higher dimensions. We now describe the method by which we aggregate buffer occupancy and throughput along a single dimension.

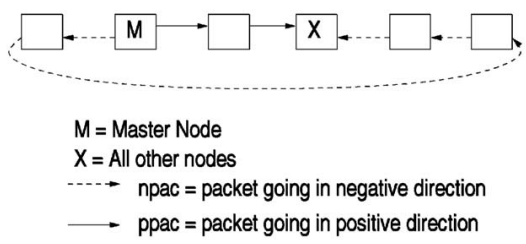

Fig. 10. Row aggregation using two metapackets. 


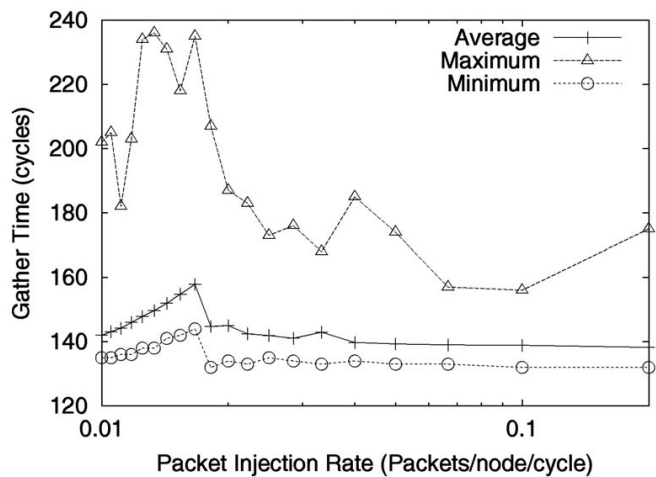

Fig. 11. Minimum, maximum, and average gather times using metapackets.

We define a $k$ th dimension row as the set of nodes which differ only in their $k$ th dimension coordinate. (In a $k$-ary $n$-cube, there are $k^{n-1}$ rows of $k$ nodes each along each dimension.) We select one master node in each row for a given dimension. This master node sends out two meta packets (ppac and npac, as shown in Fig. 10), one in each direction. One of these packets has the master node's buffer occupancy and throughput information and the other

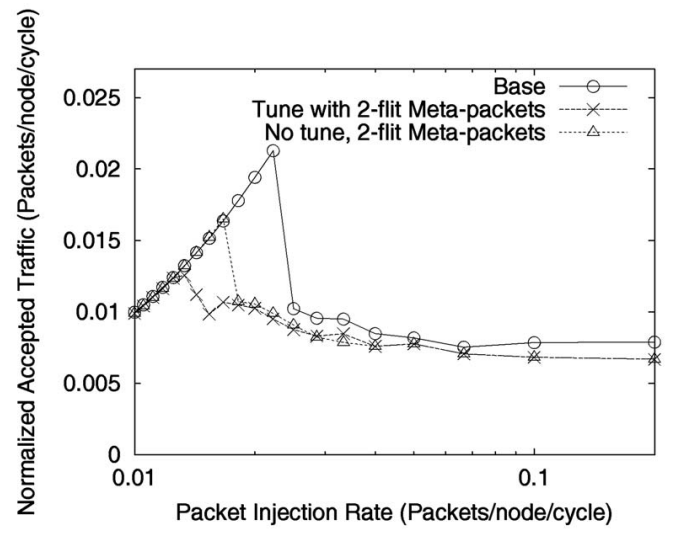

Fig. 12. Effect of metapackets and tuning on throughput.

packet is initialized to zero. These packets traverse the entire row till they are delivered back to the master node after completing a circuit. Each intermediate node modifies the metapacket by adding in its own buffer occupancy and throughput information before forwarding the node. Since each node modifies the contents of the packet before forwarding it onwards, we assess a one cycle adder-delay

\section{Deadlock Recovery}

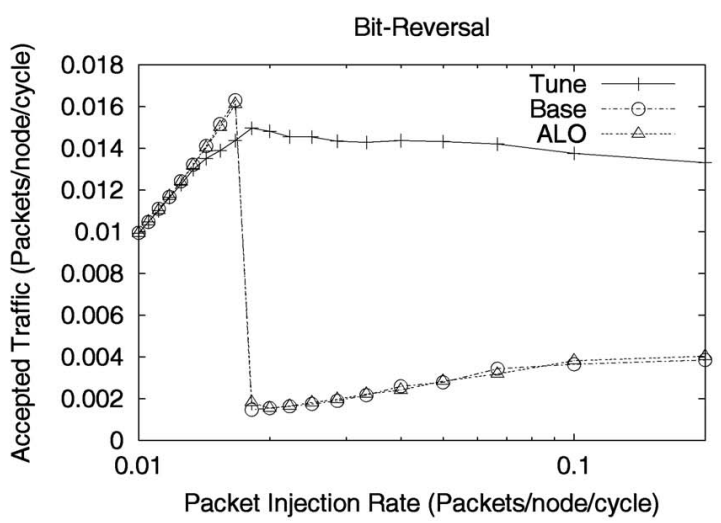

(a)

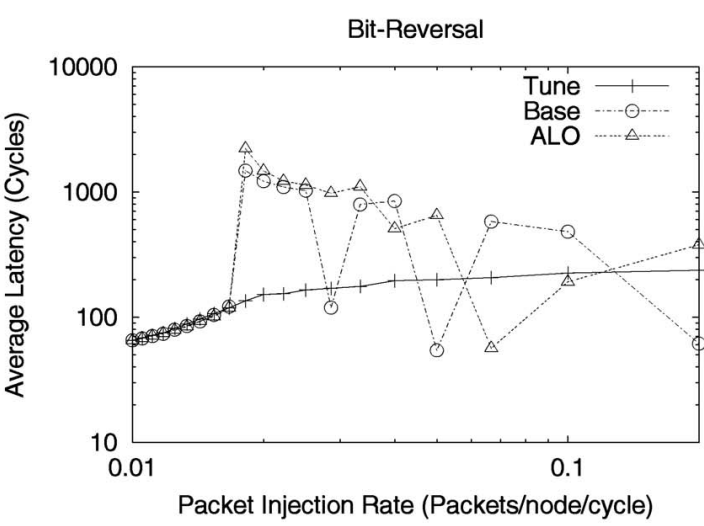

(b)

Deadlock Avoidance

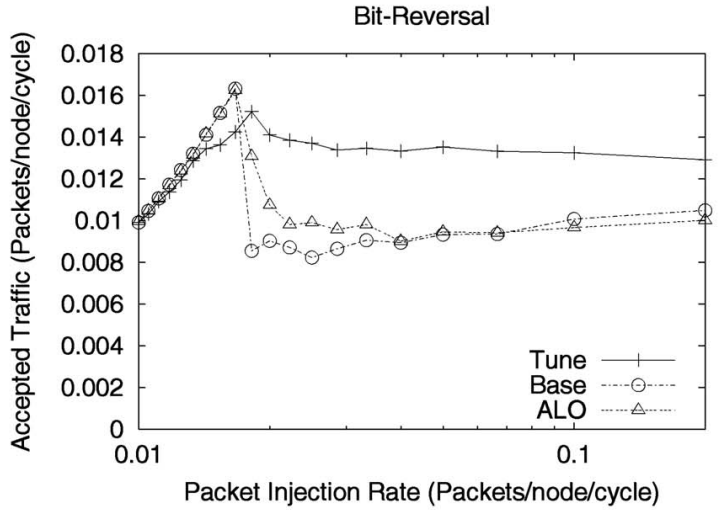

(c)

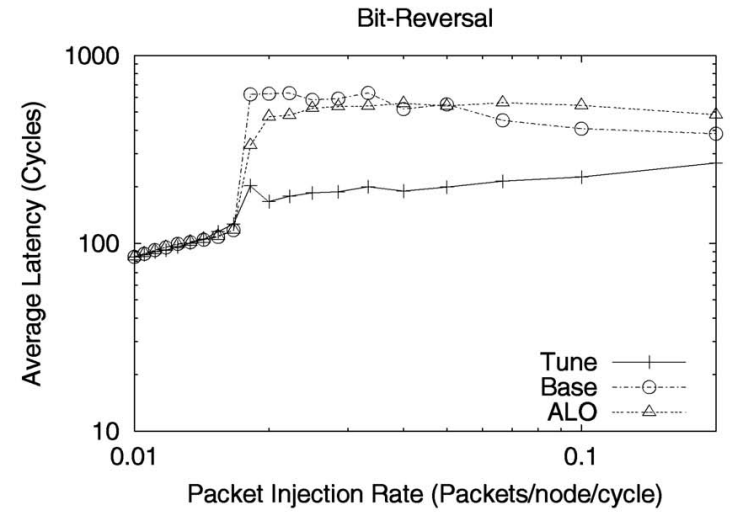

(d)

Fig. 13. Overall performance with bit-reversal traffic pattern. (a) and (c) Delivered throughput versus offered load. (b) and (d) Average latency versus offered load. 


\section{Deadlock Recovery}

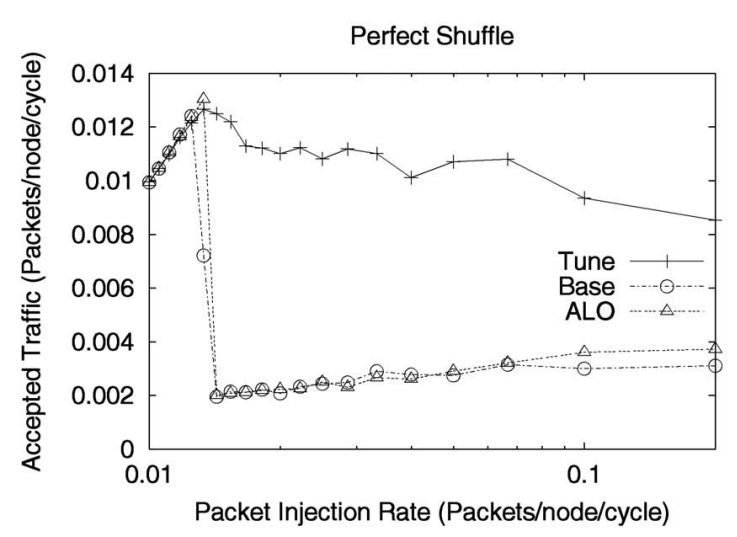

(a)

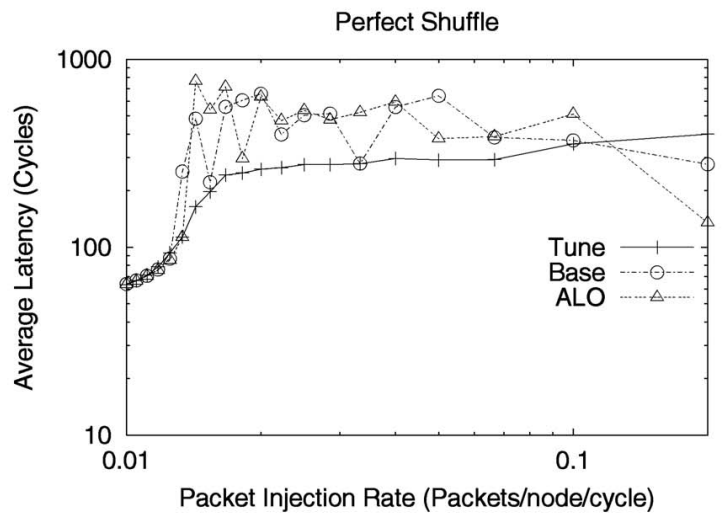

(b)

Deadlock Avoidance

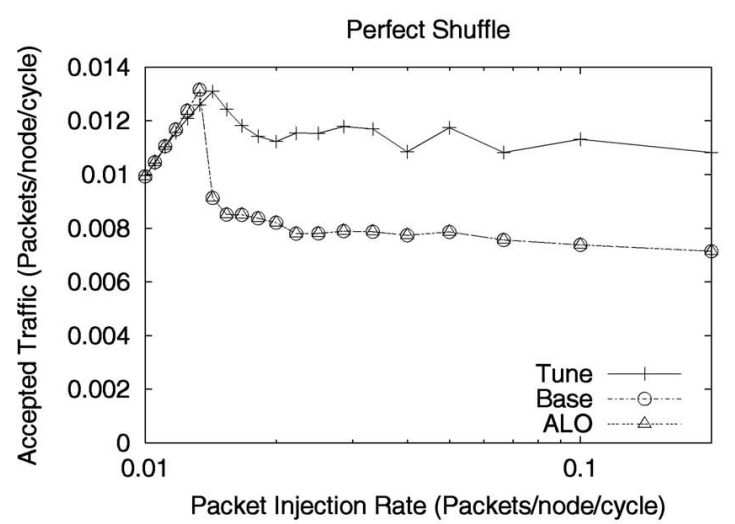

(c)

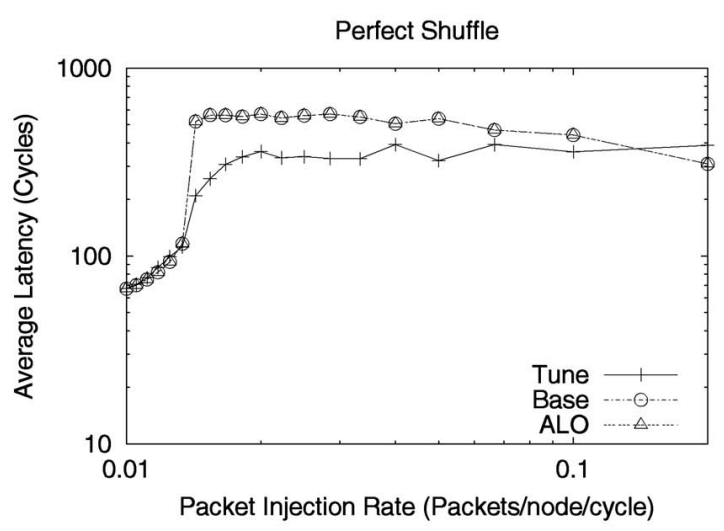

(d)

Fig. 14. Overall performance with perfect-shuffle traffic pattern. (a) and (c) Delivered throughput versus offered load. (b) and (d) Average latency versus offered load.

for the meta packets. When the two packets arrive at some node $X$ in the row, they contain the partial aggregates of information for two disjoint sections of the row (See Fig. 10). The node $X$ accumulates these partial aggregates contained in the meta packets and adds in its own local buffer occupancy and throughput information to compute the aggregate for the entire row.

We repeat this cycle for rows along the next dimension but instead of adding in local information, each node adds in the row-aggregate along the lower dimensions. After $n$ such phases, aggregation in a $k$-ary $n$-cube is complete. In the next section, we present experimental results that quantify the gather times using the information distribution schemes described above and we evaluate the performance of our congestion-control scheme using metapackets.

\subsubsection{Experimental Results}

In this experiment, we measure gather times and study the effect of varying regular data-packet load on the gather times. Fig. 11 plots maximum, minimum, and average gather times using metapacket against varying data-packet load. The $\mathrm{X}$-axis shows the applied data-packet load in packets/load/cycle and the Y-axis shows the gather times in cycles. We see that, on average, the gather time stays between 130 and 160 cycles. The maximum gather times do not exceed 300 cycles because of the higher priority accorded to the metapacket virtual channel. Further, we see that gather times drop beyond saturation loads. This is because data-packets are blocked at saturation and thus metapackets see no contention for physical links.

The next experimental result, shown in Fig. 12, illustrates the effect of using our congestion-control scheme with metapackets. Apart from the base case and our Tune configuration using meta packets, we consider another case called No tune. In the No tune configuration, we still gather the aggregate buffer occupancy and throughput information using metapackets but the gathered information is not used to throttle network packets. This allows us to isolate the effect of contention caused by meta packets and the effect of using metapacket information to throttle network packets. We see that distributing metapackets causes saturation at lighter loads. Further, using our Tune mechanism further exacerbates the situation causing saturation at lighter loads. This is partly because of the larger gather delays which causes our Tune mechanism to act on stale information. In conclusion, we find that using metapackets as the information distribution mechanism is not suitable for our self-tuned congestion control scheme. 


\section{Deadlock Recovery}

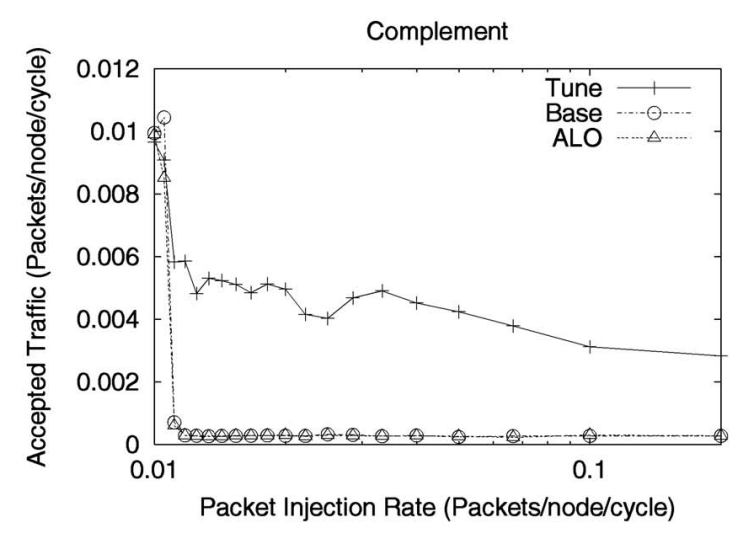

(a)

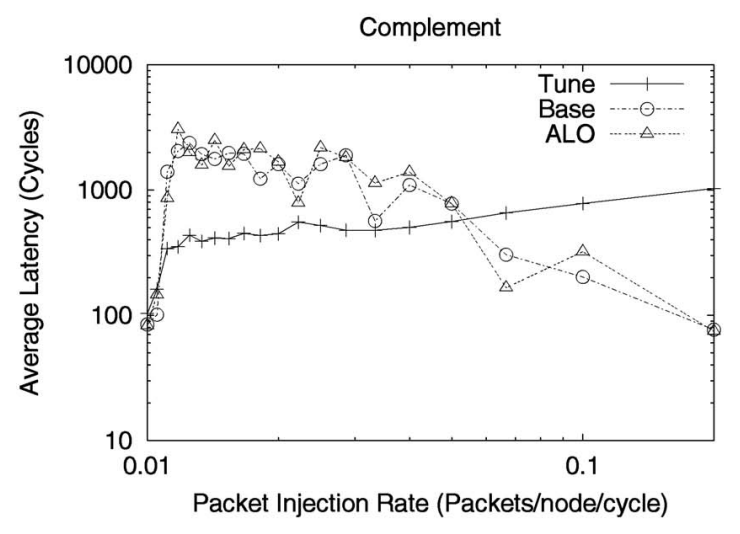

(b)

Deadlock Avoidance

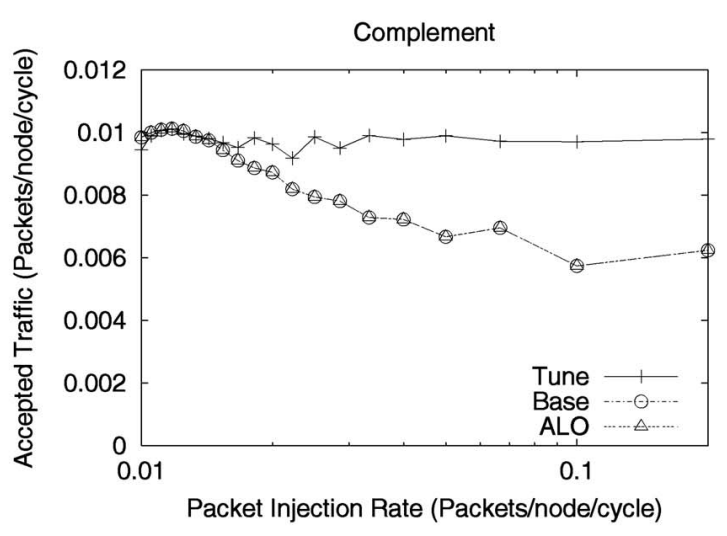

(c)

Fig. 15. Overall performance with complement traffic pattern. (a) and (c) versus offered load.

\section{Conclusion}

Interconnection network saturation, and the commensurate decrease in performance, is a widely known problem in multiprocessor networks. Limiting packet injection when the network is near saturation is a form of congestion control that can prevent such severe performance degradation. Ideal congestion control implementations provide robust performance for all offered loads and do not require any manual tuning.

The primary contribution of this paper is the development of a robust, self-tuned congestion control technique for preventing network saturation. Two key components form the basis for our proposed design. First, we use global knowledge of buffer occupancy to estimate network congestion and control packet injection. When the number of full buffers exceeds a tunable threshold, packet injection is stopped. When congestion subsides, the full buffer count drops below the threshold and packet injection restarts.

The second piece of our solution is a self-tuning mechanism that observes delivered network throughput to automatically determine appropriate threshold values. Inappropriate thresholds can either overthrottle the network, unnecessarily limiting throughput, or underthrottle and not prevent saturation. A self-tuning mechanism is important

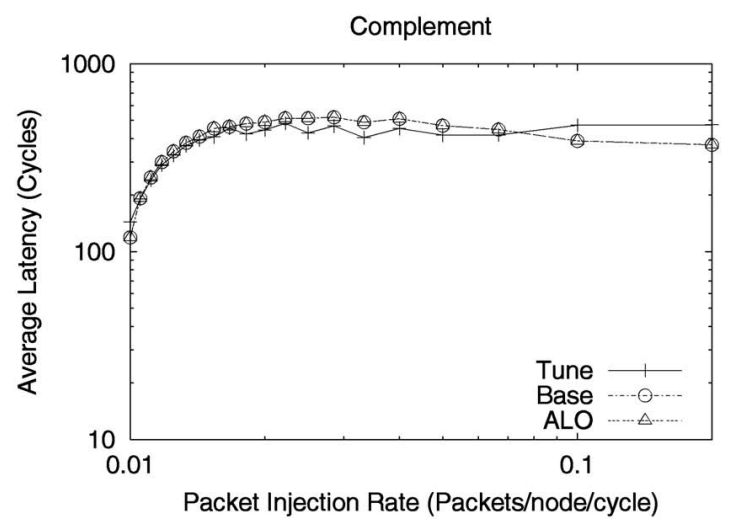

(d)

Delivered throughput versus offered load. (b) and (d) Average latency since no single threshold value provides the best performance for all communication patterns.

Using simulation, we show that our design prevents network saturation by limiting packet injection. The results also show that our technique is superior to an alternative implementation that uses local estimates of congestion because global information can detect congestion in its early stages. We show that different communication patterns require different threshold values to prevent saturation without unnecessarily limiting performance, and that our self-tuning mechanism automatically adjusts to changes in communication patterns.

\section{APPENDIX A}

Results for steady loads with bit-reversal, perfect shuffle, and complement communication patterns are shown in Figs. 13, 14, and 15.

\section{ACKNOWLEDGMENTS}

The authors would like to thank Amin Vahdat, Jeffrey Chase, Gershon Kedem, Kenneth Yocum, Joel Emer, Mark Hill, and the anonymous referees for their feedback and 
suggestions to improve this paper. They would like to thank Timothy Pinkston, Yungho Choi, and Yong Ho Song for providing the flexsim network simulator and helping to debug their simulation code. They would also like to thank Jose Duato for giving them pointers to his work on network saturation and Craig Stunkel for the pointers to the HiPPI networking standard and SPIDER router chip.

This work is supported in part by DARPA Grant DABT6398-1-0001, US National Science Foundation Grants CDA-972637, CDA-95-12356, and EIA-99-72879, Career Award MIP97-02547, Duke University, and an equipment donation through Intel Corporation's Technology for Education 2000 Program. The views and conclusions contained herein are those of the authors and should not be interpreted as representing the official policies or endorsements, either expressed or implied, of DARPA or the US Government.

\section{REFERENCES}

[1] D. Basak and D.K. Panda, "Alleviating Consumption Channel Bottleneck in Wormhole-Routed k-Ary n-Cube Systems," IEEE Trans. Parallel and Distributed Systems, vol. 9, no. 5, pp. 481-496, May 1998.

[2] E. Baydal, P. Lopez, and J. Duato, "A Simple and Efficient Mechanism to Prevent Saturation in Wormhole Networks," Proc. 14th Int'l Parallel and Distributed Processing Symp., pp. 617-622, 2000.

[3] L.S. Brakmo and L.L. Peterson, "TCP Vegas: End to End Congestion Avoidance on a Global Internet," J. Selected Areas in Comm., vol. 13, no. 8, pp. 1465-1480, Oct. 1995.

[4] W.J. Dally, "Virtual-Channel Flow Control," IEEE Trans. Parallel and Distributed Systems, vol. 3, no. 2, pp. 194-205, Feb. 1992.

[5] W.J. Dally and H. Aoki, "Deadlock-Free Adaptive Routing in Multicomputer Networks Using Virtual Channels," IEEE Trans. Parallel and Distributed Systems, vol. 4, no. 4, pp. 466-475, Apr. 1993.

[6] W.J. Dally and C.L. Seitz, "The TORUS Routing Chip," J. Distributed Computing, vol. 1, no. 3, pp. 187-196, Oct. 1986.

[7] W.J. Dally and C.L. Seitz, "Deadlock-Free Message Routing in Multiprocessor Interconnection Networks," IEEE Trans. Computers, vol. 36, no. 5, pp. 547-553, May 1987.

[8] K. Diefendorff, "Power4 Focuses on Memory Bandwidth," Microprocessor Report, vol. 13, no. 13, Oct. 1999.

[9] J. Duato, "A New Theory of Deadlock-Free Adaptive Routing in Wormhole Networks," IEEE Trans. Parallel and Distributed Systems, vol. 4, no. 12, pp. 1320-1331, Dec. 1993.

[10] J.S. Emer, "Simultaneous Multithreading: Multiplying Alpha Performance," Microprocessor Forum, Oct. 1999.

[11] S. Floyd, "TCP and Explicit Congestion Notification," ACM Computer Comm. Rev., vol. 24, no. 5, pp. 10-23, Oct. 1994.

[12] S. Floyd and V. Jacobson, "Random Early Detection Gateways for Congestion Avoidance," IEEE/ACM Trans. Networking, vol. 1, no. 4, pp. 397-413, Aug. 1993.

[13] M. Galles, "SPIDER: A High Speed Network Interconnect," IEEE Micro, vol. 17, no. 1, pp. 34-39, Jan./Feb. 1997.

[14] P.T. Gaughan and S. Yalamanchili, "Adaptive Routing Protocols for Hypercube Interconnection Networks," Computer, pp. 12-22, May 1993.

[15] HIPPI Standards Committee, "High-Performance Parallel Interface-6400 Mbit/s Physical Layer (HIPPI-6400-PH)," http:// www.hippi.org/c6400PH.html, 2003.

[16] V. Jacobson, "Congestion Avoidance and Control," Proc. ACM SIGCOMM '88 Symp., pp. 314-329, Aug. 1988.

[17] R. Jain, "Congestion Control and Traffic Management in ATM Networks: Recent Advances and a Survey," Computer Networks and ISDN Systems, Oct. 1996.

[18] P. Kermani and L. Kleinrock, "Virtual Cut-through: A New Computer Communication Switching Technique," Computer Networks, vol. 3, pp. 267-286, 1979.

[19] J.H. Kim, Z. Liu, and A.A. Chien, "Compressionless Routing: A Framework for Adaptive and Fault-Tolerant Routing," Proc. 21st Int'l Symp. Computer Architecture, Apr. 1994.
[20] K.V. Anjan and T.M. Pinkston, "An Efficient, Fully Adaptive Deadlock Recovery Scheme : Disha," Proc. 22nd Ann. Int'l Symp. Computer Architecture, pp. 201-210, June 1995.

[21] J. Laudon and D. Lenoski, "The SGI Origin: A ccNUMA Highly Scalable Server," Proc. 24th Int'l Symp. Computer Architecture, pp. 241-251, June 1997.

[22] P. Lopez, J.M. Martinez, and J. Duato, "DRIL : Dynamically Reduced Message Injection Limitation Mechanism for Wormhole Networks," Proc. Int'l Conf. Parallel Processing, pp. 535-542, Aug. 1998.

[23] P. Lopez, J.M. Martinez, J. Duato, and F. Petrini, "On the Reduction of Deadlock Frequency by Limiting Message Injection in Wormhole Networks," Proc. Parallel Computer Routing and Comm. Workshop, June 1997.

[24] L.-S. Peh and W.J. Dally, "Flit-Reservation Flow Control," Proc. Sixth Int'l Symp. High Performance Computer Architecture, pp. 73-84, Jan. 2000.

[25] G.F. Pfister and V.A. Norton, "Hot-Spot Contention and Combining in Multistage Interconnection Networks," IEEE Trans. Computers, vol. 34, no. 10, pp. 943-948, Oct. 1985.

[26] K.K. Ramakrishnan and R. Jain, "A Binary Feedback Scheme for Congestion Avoidance in Computer Networks," ACM Trans. Computer Systems, vol. 8, no. 2, pp. 158-181, 1990.

[27] D.J. Scales, K. Gharachorloo, and C.A. Thekkath, "Shasta: A Low Overhead, Software-Only Approach for Supporting Fine-Grain Shared Memory," Proc. Seventh Symp. Architectural Support for Programming Languages and Operating Systems, pp. 174-185, 1996.

[28] I. Schoinas, B. Falsafi, A.R. Lebeck, S.K. Reinhardt, J.R. Larus, and D.A. Wood, "Fine-Grain Access Control for Distributed Shared Memory," Proc. Sixth Int'l Conf. Architectural Support for Programming Languages and Operating Systems, pp. 297-306, 1994.

[29] S. Scott and G. Sohi, "The Use of Feedback in Multiprocessors and Its Application to Tree Saturation Control," IEEE Trans. Parallel and Distributed Systems, vol. 1, no. 4, pp. 385-398, Oct. 1990.

[30] S.L. Scott, "Synchronization and Communication in the T3E Multiprocessor," Proc. Seventh Int'l Conf. Architectural Support for Programming Languages and Operating Systems, pp. 26-36, Oct. 1996.

[31] A. Smai and L. Thorelli, "Global Reactive Congestion Control in Multicomputer Networks," Proc. Fifth Int'l Conf. High Performance Computing, pp. 179-186, 1998.

[32] "The Superior Multiprocessor ARchiTecture (SMART) Interconnects Group," FlexSim, Electrical Eng.-Systems Dept., Univ. of Southern California, http://www.usc.edu/dept/ceng/pinkston/ tools.html, 2003.

[33] S. Warnakulasuriya and T.M. Pinkston, "Characterization of Deadlocks in Interconnection Networks," Proc. 11th Int'l Parallel Processing Symp., Apr. 1997.

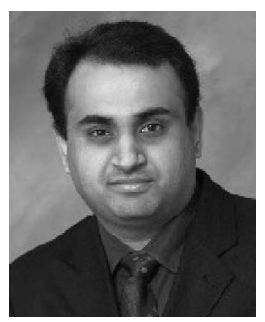

Mithuna Thottethodi received the BTech degree in computer science and engineering from the Indian Institute of Technology, Kharagpur, in 1996 and the $\mathrm{PhD}$ degree in computer science from Duke University, in 2002. He is an assistant professor of electrical and computer engineering at Purdue University. His research interests include high-performance interconnection networks, distributed microarchitectures, and multiprocessor systems. He is a member of the ACM and the IEEE. 


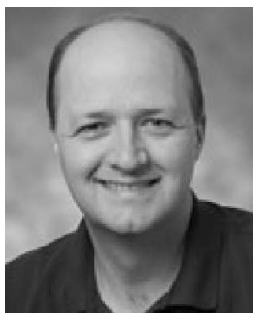

Alvin R. Lebeck received the BS degree in electrical and computer engineering, and the MS and $\mathrm{PhD}$ degree in computer science at the University of Wisconsin-Madison. He is an associate professor of computer science and of electrical and computer engineering at Duke University. His research interests include architectures for emerging nanotechnologies, highperformance microarchitectures, hardware and software techniques for improved memory hierarchy performance, multiprocessor systems, and energy efficient computing. He received the best paper award at the 31st IEEE/ACM International Symposium on Microarchitecture. He is the recipient of a 1997 the US National Science Foundation CAREER award, has received funding from the US National Science Foundation, DARPA, Intel, Compaq, Microsoft, IBM, and is a member of the ACM and a senior member of the IEEE and the IEEE Computer Society.

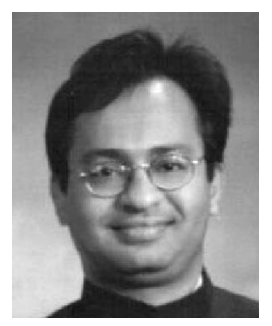

Shubhendu S. Mukherjee received the MS degree and $\mathrm{PhD}$ degree from the University of Wisconsin-Madison and the BTech degree from the Indian Institute of Technology, Kanpur. He is a senior staff hardware engineer in VSSAD, MMDC, Intel Corporation. His interests include fault-tolerant processors and high-performance interconnection networks. In the past, he has worked for Digital Equipment Corporation for 10 days and Compaq for more than three years, where he was involved with the design and development of the Alpha 21364 network architecture and fault-tolerant techniques for future Alpha processors. Currently, he codirects the FACT (Fault Aware Computing Technology) project with Dr. Joel Emer. He is a member of the IEEE.

$\triangleright$ For more information on this or any other computing topic, please visit our Digital Library at www.computer.org/publications/dlib. 